\section{Big data held to privacy laws, too}

Privacy issues around data protection often inspire overengineered responses from scientists and technologists. Yet constraints on the use of personal data mean that privacy is less about what is done with information than what is not done with it. Technology such as new algorithms may therefore be unnecessary (see S. Aftergood Nature 517, 435-436; 2015).

Technology-neutral dataprotection laws afford rights to individuals with respect to all data about them, regardless of the data source. More than 100 nations now have such dataprivacy laws, typically requiring organizations to collect personal data only for an express purpose and not to re-use those data for unrelated purposes.

If businesses come to know your habits, your purchase intentions and even your state of health through big data, then they have the same privacy responsibilities as if they had gathered that information directly by questionnaire. This is what the public expects of bigdata algorithms that are intended to supersede cumbersome and incomplete survey methods. Algorithmic wizardry is not a way to evade conventional privacy laws.

Stephen Wilson Constellation Research, Sydney, Australia. steve@constellationr.com

\section{Storm-surge models helped for Hagupit}

The Philippine government learned from shortcomings in the preparations for Typhoon Haiyan in 2013 (see R. Lejano et al. Nature 518, 35; 2015, and A. M. F. Lagmay et al. Int. J. Disaster Risk Reduct. 11, 1-12; 2015) and was able to limit the damage that last December's Typhoon Hagupit might otherwise have caused.

For example, storm surges during Haiyan extended inland by an unanticipated 2 kilometres. Specific warnings of surges of up to 5.5 metres were issued two days before Haiyan's landfall, and were broadcast on primetime television by the country's president. Despite this, the warnings proved inadequate because the variability of coastal landscapes makes it impossible to judge inundation extent on the basis of storm-surge heights alone.

Following the 2013 disaster, Project NOAH (run by the Philippine government's Department of Science and Technology) prepared stormsurge inundation maps for all its coastal provinces, modelled using high-resolution topography. These detailed maps, along with advance warning, helped to mitigate the loss of life when Hagupit's storm surges destroyed at least 1,800 homes at the end of 2014 . Alfredo Mahar Lagmay National Institute of Geological Sciences, University of the Philippines Diliman, Quezon City, the Philippines. Norman Kerle University of Twente, Enschede, the Netherlands. mlagmay@nigs.upd.edu.ph

\section{Leadership training for African scientists}

The Africa Science Leadership Program, launched on 2 March, is the first of its kind in the developing world (see www. up.ac.za/aslp). It will train researchers to lead complex scientific initiatives across disciplines and sectors, helping them to compete in global knowledge production.

A handful of institutions in Canada and the United States offer useful advanced leadership training for researchers (see, for example, M. Kvaskoff and S. D. McKay Nature 506, 159; 2014) The need for such programmes is more desperate in developing countries if they are to avoid falling further behind in an interconnected and competitive world (B. Slippers et al. S. Afr. J. Sci. 111, a0093; 2015).

The African programme will create nuclei for leadership development on the continent. Small groups of mid-career scientists will participate in the year-long training, which includes two multi-day sessions, ongoing peer support and mentoring in-between.

Bernard Slippers University of Pretoria, South Africa.

Eva Alisic Monash University, Australia.

bernard.slippers@fabi.up.ac.za

\section{Is there fame bias in editorial choice?}

Nature's Correspondence items are reviewed only by the editors (see go.nature.com/cmchno). To investigate whether editorial bias towards internationally renowned correspondents might be at play in selecting candidates for publication, we analysed the scientific status of Correspondence authors published in 2014.

We used the following pointers to gauge author reputation: faculty member in one of the world's top 100 universities (as listed in the Times Higher Education World University Rankings; see go.nature. com/bhgfxd); authorship of Nature or Science publications; high $h$ index. We classed correspondents as established scholars if they fulfilled any or all of these criteria.

The number of letters published in Correspondence in 2014 was 239, each with one 'corresponding author' responsible for submission and communication with the editors. We found that $54 \%$ of these authors met some or all of our criteria. Some 13\% of authors came from the developing world.

Within the limitations of our 'fame factors' (for example, some correspondents were well known but from outside academia), we infer that scientific celebrity of the primary correspondent does not notably influence the selection of letters for publication.

Omid Mahian ${ }^{\star}$ Ferdowsi University of Mashhad, Mashhad, Iran. omid.mahian@gmail.com ${ }^{*}$ On behalf of 4 correspondents (see go.nature.com/rpntpk for full list).

\section{Build reward system for ace technicians}

Technical staff are crucial to the smooth running of a research laboratory (see Nature 517, 528; 2015). As largely unsung heroes, they warrant rewards beyond praise and salary increases.

A senior technician's duties cover, among other functions, safety, finance, ordering and repairs. Technicians maintain stocks of essential reagents and keep facilities such as databases up to date. They carry out specialized experiments and train staff in methodology.

Rewards for such highly skilled technicians might include setting up a fund to keep them employed during times of financial constraint. Our technicians also share the right of graduate students and postdocs to attend one conference of their choice every year. Some are co-authors on scientific publications and present work at meetings. After 16 years in our lab, one technician has 110 publications to his credit, as well as more than 3,000 lifetime citations and an $h$ index of 33 . Eleftherios P. Diamandis Mount Sinai Hospital and University Health Network, Toronto; and University of Toronto, Canada. ediamandis@mtsinai.on.ca

CORRECTION

In the print version of the Outlook article 'The toxic side of rice' (Nature 514, S62-S63; 2014), reference 3 originally cited the wrong study. It has now been corrected online. 